\title{
Voluntades Anticipadas vs. Instrucciones Previas o Testamento Vital en Atención Primaria de Salud
}

\author{
a Médico de Familia. CS Pío XII. \\ Ciudad Real.
}

Correspondencia:

Francisco Angora Mazuecos. Gerencia AP de Ciudad Real, Avda Pío XII s/n,

13002-Ciudad Real. E-mail: fangoram@sescam.jccm.es

Recibido el 4 de septiembre de 2008.

Aceptado para su publicación el 21 de septiembre de 2008.

\author{
Francisco Angora Mazuecos ${ }^{a}$
}

\section{RESUMEN}

Hipótesis y Objetivo. La Ley reconoce los derechos fundamentales de los pacientes terminales, que pueden ejercer Declaración de Voluntades Anticipadas (DVA) como autentica planificación que respeta el sistema de valores personales del paciente al poder decidir morir dignamente, pero escoger en libertad requiere información clínica sobre las opciones posibles. Si actualmente pocas personas ejercen DVA, es debido a que aún no se dan las condiciones optimas que demanda la legislación sobre voluntades anticipadas (VA). El objetivo principal del estudio ha sido descubrir conocimientos sobre: prolongación de la vida, derecho legal y moral a elegir una muerte digna, limitación del esfuerzo terapéutico (LET) y existencia en Castilla La Mancha de la posibilidad de ejercer DVA.

Diseño. Estudio de intervención primaria, por muestreo consecutivo sobre pacientes de 65 y más años, emplazado en APS; mediante un "curso de introducción informativa sobre VA" y "encuesta sobre conocimientos e impresiones de VA", previos y posteriores al curso.

Emplazamiento. Centro de Salud urbano de Ciudad Real capital.

Mediciones principales. Variables sociodemográficas (edad, sexo, vivienda, convivencia, trabajo principal antes de jubilarse). Conocimientos e impresiones sobre VA y DVA, antes y después del curso. Resultados. Mayoritariamente viven en domicilio propio, tienen estudios elementales, y dedicación previa de amas de casa, funcionarios, técnicos y peones. Previo a la sesión el $60 \%$ desconocía las VA y su posibilidad de ejercer declaración. Encuestados y familiares vivirían más tranquilos declarando VA. Quieren registrar sus preferencias en la historia de APS. Tras el curso, la mayoría tiene claras las limitaciones legales, profesionales y morales de las VA. El 70\% optaría por ejercer DVA ante funcionarios del registro de VA (Delegación de Sanidad).

Conclusiones. Los mayores saben poco de VA (situación expectante). El personal y el ámbito de la APS resultan idóneos para informar-asesorar sobre VA y DVA. Procedería ampliar el estudio a otras franjas etarias.

Palabras clave. Directivas Anticipadas, Atención Primaria de Salud.

\section{ABSTRACT}

Early Wills vs. Previous Instructions or Living Wills in Primary Care

Hypothesis and objective. The legal system recognizes the fundamental rights of terminal patients or dying ones who can use a Declaration of Early Wills (DEW), that corresponds to a certified planning of treatments which respects the system of personal values of the patient, who can decide to die with dignity. However, a free choice requires clinical information about the possible options available. Nowadays, the reason why few people use DEW is that most of the legally required conditions are not fulfilled. The main objective here is to find information about the prolongation of life, legal and moral right to die with dignity, limitation of therapeutic effort (LTE) and the possibility of using DEW in Castilla La Mancha.

Design. Study of primary intervention using a consecutive sample of patients over 65, from a PC setting, using "an informative course that gives an introduction to the EW" and "a survey of knowledge and opinions about EW", before and after the course.

Setting. Urban Health Centre of Ciudad Real.

Main Variables. Socio-demographic variables (age, gender, house, cohabitation, main job before retirement). Knowledge about and impressions of EW and DEW, before and after the course.

Main findings. The vast majority live in their own houses, have elementary studies and worked as housewives, civil servants, technicians or unskilled labourers. Before the session, $60 \%$ did not know anything about EW and the possibility of declaring them. People participating in the survey, and relatives, would feel more reassured if they could declare EW. They would like to register their preferences in their PC records. After the course, the vast majority have a better understanding of the legal, professional and moral limitations of the EW. A total of $70 \%$ would sign DEW before civil servants of the EW registry (Health office).

Conclusion. Individuals have little information about EW at present. Staff from PC are the most suitable people to inform patients about them and should give the patients a real freedom of choice. This study should be broadened to include people of different ages.

Keywords. Advance Directives, Primary Health Care. 


\section{INTRODUCCIÓN}

Ante los recientes ataques a la sanidad pública española en general, y madrileña en particular, con la sedación paliativa como excusa de mala praxis de eutanasia activa encubierta, cuya reparación moral va a ser de facto insuficiente, aporto un enfoque distinto al hospitalocentrista habitual, y lo hago desde la APS, por la integralidad asistencial y sus posibilidades anticipatorias preventivas, educacionales e informativas.

Es conocida la Recomendación 1418 (1999), de 25 de Junio, de la Asamblea Parlamentaria del Consejo de Europa $^{1}$ a sus 41 estados miembros (España incluida), sobre los derechos fundamentales que derivan de los pacientes terminales o moribundos, que se ven amenazados por diversos factores como el uso desproporcionado de medios técnicos o la continuación o inicio de un tratamiento sin consentimiento del paciente, con la consecuente prolongación del proceso de muerte contra la voluntad del enfermo terminal, sin que este pueda influir en el recorrido de este proceso del morir. Es acorde con el art. 9 del Convenio de Oviedo $^{2}$, sobre los deseos expresados anteriormente por el paciente, para la protección de los derechos humanos con respecto a las aplicaciones de la biología y la medicina.

Según el informe publicado por el Hasting Center $^{3}$ en 1996, las cuatro metas de la medicina en la actualidad son: prevenir la enfermedad y las lesiones y promocionar la salud, aliviar el dolor y sufrimiento de enfermedades y dolencias, asistir y curar (cuidar a los que no pueden ser curados) y evitar la muerte prematura y velar por una muerte en paz. Sin embargo, la experiencia en países que vienen aplicando desde hace algunos años VA es, en general, poco optimista. En Europa, aunque teóricamente deberían ser ya aceptadas por la población, apenas se han asimilado, y pocos ejercen declaración de las mismas, siendo aventurado sacar conclusiones aún sobre su implantación.

Con todo, ciudadanos y profesionales sanitarios coinciden en el interés de este derecho, especialmente en pacientes con demencia o no capaces llegada la fase terminal o irreversible de la vida, ya que en algunas de estas situaciones el médico debería anticipar el pronóstico para dar tiempo a que la voluntad se refleje de algún modo, antes de que el paciente sea no capaz, y deberíamos asumir que la DVA y la figura del representante (interlocutor único) constituyen la prolongación de la autonomía del paciente, favoreciendo su implantación y desarrollo.

Distintos autores coinciden que el ámbito de la APS es el más favorable para introducir al paciente en los aspectos concernientes a las VA, pero la tozudez de la realidad constata que no está siendo así, y en tal sentido el reciente estudio de Nekane Lauzirika ${ }^{4}$ concluye, entre otros datos, que el $83 \%$ de los profesionales sanitarios no tienen suficiente información para abordar el tema de las VA respecto a sus pacientes.

EI MF pretende abarcar todas las materias relacionadas con la salud humana en los aspectos biológico, psíquico y social, de forma lineal y de por vida, individualizando la atención integral de la persona, asistiendo a domicilio y en el centro de salud (CS), por demanda del propio paciente, de forma programada o por vía urgente; siendo el receptor y responsable principal, que coordina, "tamiza", recompone y decide sobre la oportunidad de las diferentes propuestas emitidas por otras especialidades médicas, en general hospitalarias, pero también privadas o concertadas. Se trata, en la practica, de una misión que de manera no siempre consciente se desarrolla en exclusiva en el marco de la APS, cual es la denominada "prevención cuaternaria", ante el excesivo intervencionismo dominante en una sociedad insaciable de tecnología y de opiniones "expertas", de la mano de una paradójica inseguridad, miedo e indefensión culturales que alimentan mediáticamente la industria tecnológico-farmacéuticosanitaria privada y que sazonan las políticas cortoplacistas irresponsables. En tan generosa oferta radica la grandeza de la Medicina Familiar y Comunitaria (MFyC) y su propio talón de Aquiles, por la vasta responsabilidad que asume y al estar sometida a los constantes y rápidos cambios de los pilares de la ciencia médica, considerados hasta hace poco mas de un siglo casi inamovibles.

La fructífera y tradicional relación médico-paciente, basada en el modelo paternalista del primero, va dejando paso en las últimas décadas al predominio de la autonomía del segundo ${ }^{5,6}$, de modo que actualmente la regulación de la toma de decisiones en el proceso clínico se basa de manera fundamental en el respeto a la dignidad humana, la autonomía de la voluntad y a las resoluciones libremente adoptadas por las personas en el ámbito de su salud (art. 2 de la Ley 41/2002 o Ley Básica Reguladora de la Autonomía del paciente y de Derechos y Deberes en materia de Información y Documentación Clínica ${ }^{5}$ ).

Pero, para que los ciudadanos en general puedan escoger en libertad dentro de las opciones posibles que la ciencia médica les ofrece al respecto, incluso la de no someterse a ningún tratamiento ni intervención o a alguno/s concretos, resulta preceptiva la información clínica oportuna, clara y no sesgada, siendo el proceso de información algo más que un mero formalismo. Así, tal y como nos recuerda la STC 132/1989 de 18 de junio, lo anterior encuentra fundamento y apoyo en la misma Constitución Española, en la exaltación de la 


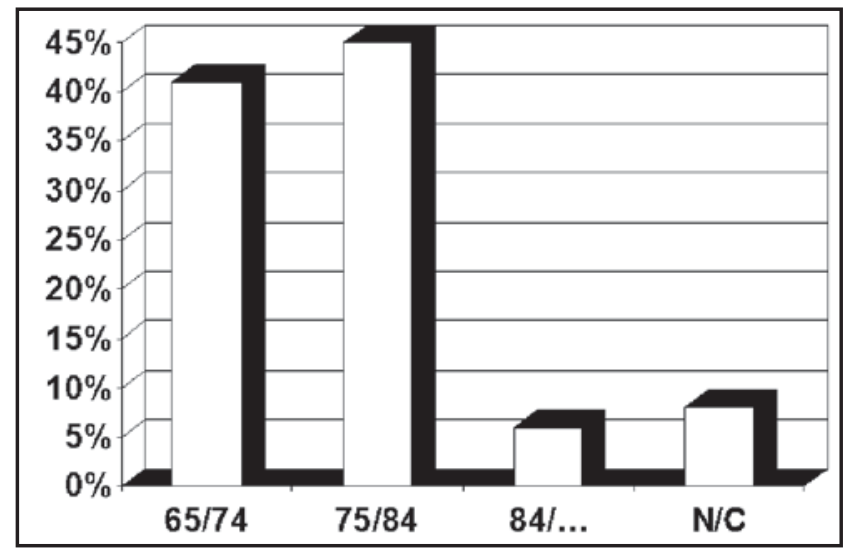

Figura 1. Participación por bloques etarios.

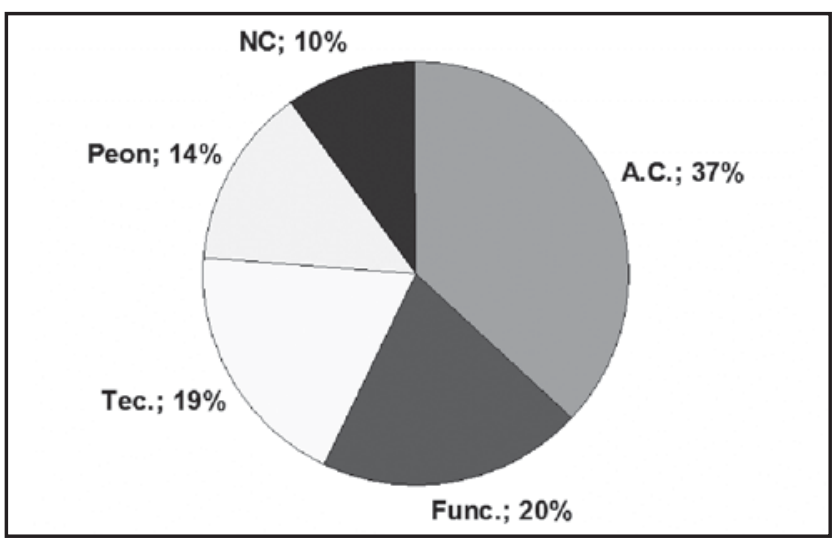

Figura 2. Trabajos previos a la jubilación. A:C.: ama de casa, Tec.: técnico titulado, Func.: funcionario, NC: no contesta

dignidad de la persona que se consagra en su art. 1.1 y el art. 9.2, reconociendo la autonomía del individuo para elegir entre las diversas opciones vitales que se presenten, de acuerdo con sus propios intereses y preferencias $^{7,8,9}$.

Desde el ámbito de la APS, donde desarrollo mi labor como médico de familia, observo que con frecuencia ignoramos la calidad de vida de los enfermos terminales, la soledad a la que se ven sometidos, su sufrimiento y el de sus familiares, así como el sufrimiento del personal sanitario que los trata ${ }^{9}$, habitualmente no considerado en la justa medida. Una vez reabierto el debate profesional y social sobre los derechos de las personas al respecto y sobre quién decide, si no lo ha hecho previamente a la perdida de la capacidad el propio interesado $2,10,11$, entendemos, con un enfoque pretendidamente ético, que lo legislado sobre VA respeta el sistema de valores de muchas personas que no desean aguantar un final de vida que no consideran digno, o que no admiten que para sobrevivir deban someterse a técnicas diagnosticas y/o terapéuticas contrarias a su voluntad, moral o creencias, o en el mejor de los casos actuaciones fútiles.

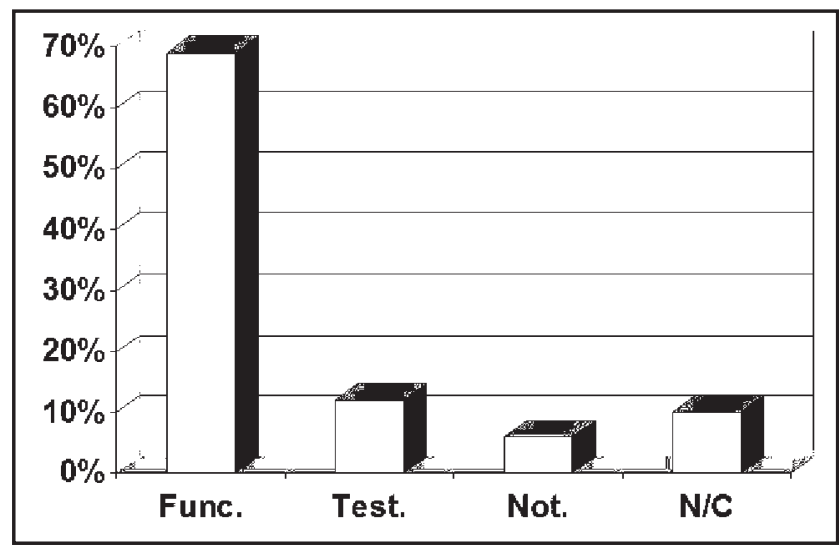

Figura 3. Elección modalidades para declarar V.A.

Asumimos que las VA constituyen no solo una oportunidad de limitar actuaciones fútiles en ciertas situaciones terminales o de inconsciencia irreversible, sino además una autentica planificación anticipada de tratamientos, que pretende satisfacer las preferencias del paciente.

En todo caso, en la práctica diaria, resulta frecuente que la disyuntiva entre ciencia y valores, como afirma Santos Unamuno ${ }^{12}$, no permita a los profesionales afrontar los problemas bioéticos en toda su complejidad, dado que el imperativo tecnológico dominante en la sociedad actual lleva a los médicos a hacer con el paciente "todo lo que puede hacerse", sin pensar en "lo que debe hacerse". Y teniendo las VA como finalidad, para que el paciente muera a su debido tiempo y sin sufrimientos innecesarios, se acepta desde la bioética, como afirma Giacomo Perico ${ }^{13}$, que el paciente debe vivir porque la vida es un don, pero considerando el "principio del doble efecto", por el que se acepta el uso de medidas que alivien el sufrimiento, aunque tengan como efecto secundario acortar la vida del paciente, siempre que entre ambos efectos haya una proporcionalidad razonable que compense.

Revisadas las posibilidades que recoge la DVA (expresión de valores personales, instrucciones sobre cuidados y tratamientos, posible nombramiento de un representante y decisión sobre sus órganos y/o cuerpo) y considerando los previos postulados, sostengo la hipótesis de que aún no se ha producido en nuestro medio la deseable adaptación al cambio social, personal y profesional que demanda la legislación al respecto, la española (Ley básica 41/2002, de 14 de noviembre, arts. 5, 9 y 11) y la autonómica de Castilla-La Mancha (Ley 6/2005, de 7 de julio, D.O.C.M. no 141, de 15 de julio), aportando ambas la "carga ética formal" de la reciente legislación sanitaria ${ }^{14}$.

En EEUU surge a mediados del siglo XX el término "advance directives", coincidiendo con el debate so- 
bre la eutanasia, y en 1967 se acuña el de Living Will (testamento vital), con el objetivo de auto-controlar la duración de la vida y las condiciones del morir. En 1976 se promulga en California la Ley de muerte natural y en 1991 se aprueba la Ley Federal de Autodeterminación del paciente, con la finalidad principal de resolver los conflictos éticos derivados de la retirada, o no instauración de tratamientos en situaciones irreversibles. Posteriormente, los esfuerzos están siendo dirigidos a perfeccionar las directivas anticipadas, contempladas como la extensión del consentimiento informado a las situaciones de incapacidad, para decidir sobre los tratamientos según las preferencias del paciente en aquellas situaciones permitidas. Así, en España se han ido aportando documentos:

- En 1986, el "testamento vital" de la Asociación Derecho a Morir Dignamente (DMD) ${ }^{15}$, en el que el declarante pide que se le respete la opinión respecto a su propio cuerpo, en función de los derechos de la persona sobre su vida.

- En 1986, "testamento vital" de la Conferencia Episcopal Española ${ }^{16}$ en función de los valores religiosos de la iglesia católica, pidiendo los oportunos tratamientos paliativos del sufrimiento, aunque rechaza el encarnizamiento terapéutico y la eutanasia activa.

- En 1996, la "Directriz o exoneración médica preliminar ${ }^{17}$ de los Testigos de Jehová" da los fundamentos religiosos para rechazar las trasfusiones de sangre y hemoderivados.

- En España, la primera regulación jurídica sobre VA surge en Cataluña (Ley 21/2000), sobre los derechos de información concernientes a la salud, a la autonomía del paciente y a la documentación clínica, emitiendo el comité de bioética de Cataluña, en colaboración con el Colegio de Notarios, su propio modelo de testamento vital ${ }^{18}$.

- Modelo de instrucciones previas de la Organización Medica Colegial ${ }^{19}$.

- A la iniciativa legal catalana han sucedido las de otras Autonomías, encontrándose estas en fases muy diferentes de su desarrollo ${ }^{20}$.

- La Regulación Estatal, de 15 de noviembre de 2002, Ley 41/2002, Básica Reguladora de la Autonomía del Paciente y de Derechos y Obligaciones en materia de Información y Documentación Clínica ${ }^{5}$, en su artículo 11 dice sobre las denominadas "instrucciones previas" (IP): a) Una persona mayor de edad, capaz y libre, manifiesta anticipadamente su voluntad, con objeto de que ésta se cumpla en el momento en que llegue a situaciones en cuyas circunstancias no sea capaz de expresarlos personalmente, sobre los cuidados y el tratamiento de su salud o, una vez llegado el fallecimiento, sobre el destino de su cuerpo o de los órganos del mismo, pudiendo designar un representante, que llegado el caso haría de interlocutor para procurar el cumplimiento de dichas IP. b) Cada Servicio Autonómico de Salud regulará el procedimiento adecuado para que, llegado el caso, se garantice el cumplimiento de las IP, que deberán constar siempre por escrito. c) No serán aplicadas las IP contrarias al ordenamiento jurídico, a la "lex artix", ni las que no correspondan con el supuesto de hecho que el interesado haya previsto en el momento de manifestarlas. En la $\mathrm{HC}$ del paciente quedará constancia de las IP. d) Las IP podrán revocarse libremente en cualquier momento dejando constancia escrita. e) Con el fin de asegurar la eficacia en todo el territorio nacional de las IP formalizadas en las diferentes Autonomías, se creará en el Ministerio de Sanidad y Consumo, el Registro Nacional de IP.

Los objetivos del estudio son: 1) Descubrir el grado de conocimiento (introspección) sobre posibilidades médicas de prolongar la vida en condiciones extremas, derecho moral y legal a una muerte digna, limitación del esfuerzo terapéutico (LET) y diagnostico (LED), eutanasia, posibilidad en CLM de declarar y registrar VA. 2) Divulgar activamente la guía de la Junta de Comunidades de CLM sobre VA y la posibilidad de DVA y RDVA entre los usuarios de la APS, así como entroncar tal divulgación en las Actividades de Educación para la Salud (EpS). 3) Registrar (autorizado) la existencia de DVA, en la HC de AP. 4) Conocer el grado de apoyo de los ciudadanos a la planificación por adelantado de los cuidados a prestar, o evitar en su caso.

\section{PACIENTES Y MÉTODO}

Mediante un muestreo consecutivo $(n=51)$, se seleccionó a los pacientes de 65 y más años que requirieron asistencia en APS entre el 1 de junio de 2007 y el 31 de agosto de 2007 y que habían sido previamente evaluados mediante test psicométrico en un periodo no superior a 6 meses. Se trata de un estudio de intervención de tipo primario en el que no existe asignación aleatoria, con cita personal o telefónica para realizar en las dependencias del propio C.S. el citado curso y la encuesta sobre VA.

El curso de "Introducción Informativa sobre VA" fue diseñado para ser dirigido a personas de 65 y más años vinculadas a los cupos de APS del C.S. Pío XII de Ciudad Real (red pública del SESCAM), incluyendo la administración de una encuesta voluntaria y anónima sobre conocimientos, antes y después de la sesión, y sobre el interés y oportunidad de la misma. 
El manual de procedimientos incluyó: diseño del curso de "Introducción Informativa sobre VA", diseño de la encuesta con prueba basal de validación y sistemas de control de calidad, identificación de las personas a informar y encuestar, realización de la encuesta y tratamiento estadístico y exposición de resultados, discusión y conclusiones.

Definiciones operativas de las condiciones del estudio: 1) Identificación por número de encuesta y fecha de realización. 2) Criterios de inclusión (edad de 65 ó más años, test psicométrico reciente y/o valoración clínica de no alteración cognitiva y residencia en Ciudad Real capital. 3) Criterios de exclusión (negarse a participar, alteración severa de los sentidos de la vista y/o audición, no cumplir los criterios de inclusión). 4). Conformidad para participar en el estudio, dirigido a difundir información relativa a VA, así como a la exploración del grado de conocimientos sobre el tema, abordado de forma anónima y respetando en todo caso la individualidad y privacidad en los sistemas de información.

El desarrollo del curso se estructuró de la forma siguiente: 1) Introducción, exposición oral y justificación. 2) Exposición estructurada, con apoyo logístico mediante Power point. 3) Entrega personalizada de material ad hoc, fundamentalmente los modelos informativos de la propia JCC de CLM, que incluyen todos los documentos oficiales para la DVA, y de los modelos documentales emitidos en su día por la Asociación Derecho a Morir Dignamente (DMD), la Conferencia Episcopal Española (CEE), el grupo de trabajo impulsado por el Comité de Bioética de Cataluña y la Directriz o Exoneración Médica de los Testigos de Jehová. 4) Ejemplo de cumplimentación de la encuesta. 5) Turno libre de opiniones.

\section{RESULTADOS}

Los resultados obtenidos fueron:

- Predominio no significativo de asistencia y cumplimentación de la encuesta por parte de las mujeres, sobre 51 participantes.

- Participación por grupos de edad: 65-74 años (41\%), 75-84 años (45\%) y de 85 años en adelante (6\%) (figura 1).

- $\quad$ El 37\% tiene estudios primarios, 22\% lee y escribe, $20 \%$ son bachilleres, $6 \%$ diplomados o licenciados, $6 \%$ no tienen ningún estudio y $10 \%$ no contestan.

- Trabajo antes de jubilados: $37 \%$ ama de casa, $20 \%$ funcionario (80\% hombres), $19 \%$ técnico titulado, $13 \%$ peón manual y $10 \% \mathrm{n} / \mathrm{c}$ (figura 2 ).

- Vive en casa o piso propio el $69 \%$, con hijos o familiares el $13 \%$, en residencias el $2 \%$ y el $10 \%$ no contesta.

- Convive con la pareja el 53\% (2/3 son hombres), vive solo el $22 \%$ ( $2 / 3$ son mujeres), otros tipos de convivencia el $13 \%$ y no contesta el $10 \%$.

- Dice participar libremente el 96\%.

- Conocimientos previos sobre VA ("enterados") el $47 \%$ (2/3 son mujeres) y de que ya puede efectuarse DVA en CLM DVA el 39\%.

- Estarían más tranquilos tras hacer DVA en el 88\% de los casos y también lo estarían sus familias en el $84 \%$.

- De las tres modalidades propuestas en nuestra autonomía para cumplimentar la DVA, el $69 \%$ se inclina por la opción "ante el funcionario del registro de DVA", el $12 \%$ "ante tres testigos" y el $6 \%$ "ante notario", y no contesta el 10\% (figura 3).

- En caso de hacer DVA, se lo comunicaría a su médico de familia para hacerlo constar en la $\mathrm{HC}$ de AP el $82 \%$ (ninguno en desacuerdo).

- Queda claro al 78\% que las VA solo se tienen en cuenta en situaciones limite en que el paciente no puede expresar su voluntad, persistiendo dudas tras el curso en el $13 \%$ de encuestados.

- $\quad$ El $86 \%$ entiende bien que no se aplicaran en ningún caso procedimientos ilegales o contraindicados.

- Curso interesante para el $90 \%$, no contesta el $8 \%$, a un participante le ha dejado igual y poco-nada interesante a ninguno.

- Al ítem abierto a "manifestar algo libremente" responde el $19.60 \%$ (10 participantes), cuyas opiniones trascribo literalmente: "de momento no lo he pensado y el tiempo y la edad dispondrán", "si, se puede usar la libertad humana como don basado en sus creencias", "deseo no ser enchufada a ninguna máquina ni prolongar vida sin necesidad", "ánimo, me parece estupendo el curso", "si no tengo cura no me pongan tratamientos ni máquinas por prolongar la vida", "estaría bien que la eutanasia fuera legal", "la reunión por mi parte me ha dejado satisfecho y ha sido amena sobre la declaración a ( $i$ ?) decidir", "con lo que ahora sabemos da que pensar", "como nos ha dicho, para elegir con libertad primero nos tienen que informar", "me ha gustado que cuenten con los mayores como yo, muchas gracias".

\section{DISCUSIÓN}

Contando con el asumido sesgo muestral y del tratamiento estadístico básico, la alta asistencia de los invitados, cercana al $90 \%$, la cumplimentación de la encuesta por todos los asistentes, de los que al $90 \%$ les pareció interesante, la riqueza de las aportaciones en el ítem abierto y en el turno de palabra, y especial- 
mente el resultado de la encuesta, abren la puerta a la difusión generalizada de este tipo de información, sobre el derecho y las posibilidades de los castellanomanchegos mayores de edad de ejercer la DVA. Deberá llevarse a cabo utilizando para cada franja etaria a los profesionales y medios que se revelen como más eficaces. Para el caso de personas de 65 y más años parece probado que debería estar a cargo de los profesionales de APS, para ofertar la cobertura de una necesidad aparentemente no demandada, posiblemente por falta de información adecuada, especifica y oportuna.

Tal difusión requiere de la previa concienciación, información y formación al respecto de los propios profesionales, si no la tuviesen, y se enmarcaría como una actividad más de la EpS en formato charla, sobre todo a través de la entrevista clínica, evitando sensacionalismos como refiere D. Gracia ${ }^{21}$. Al respecto y desde el principialismo quiero recordar varias reflexiones notorias en el mundo de la bioética:

- Respetar la autonomía de las personas pertenece al principio de no maleficencia y es por tanto exigible, ética de mínimos, pero...

- Facilitar información para que las personas puedan participar en la toma de decisiones es algo deseable aunque no obligatorio ni exigible, y que pertenece al principio de beneficencia, siendo un marcador de excelencia en la practica profesional.

Del controvertido tema de las VA, su declaración (DVA) y posible registro (RDVA), se sabe poco en general entre la población española y en particular en la de nuestro estudio, sin embargo, la convocatoria sorprendentemente resultó ser un gancho atractivo, acudiendo casi todos los invitados al curso organizado sobre VA, que fue considerado interesante por el noventa por ciento de los participantes.

Queda pues abierta la posibilidad de ofertar la cobertura de una necesidad aparentemente no demandada mediante difusión a través de los mecanismos propios de la entrevista clínica y/o de cursos diseñados al respecto, con la información clínica suficiente que permita elegir libremente, en el derecho fundamental de autonomía de cada persona.

Queda probada, provisionalmente, la idoneidad de que, al respecto, lleven la iniciativa los profesionales de APS, recibiendo estos la oportuna información-formación que los conciencie en la búsqueda del ideal profesional de excelencia.

Seria necesario ampliar el estudio a una muestra más representativa, así como a otras franjas etarias. En- tiendo que la información sobre VA es inherente a la propia relación del personal de APS con el paciente y que no debe ser entendida como una tarea más de la cartera de servicios.

\section{BIBLIOGRAFÍA}

1. Asamblea Parlamentaria del Consejo de Europa. Protección de los derechos humanos y de la dignidad de los enfermos terminales y moribundos. 25-junio-1999.

2. Consejo de Europa: Convenio para la protección de los Derechos Humanos y la Dignidad del ser humano, con respecto a las aplicaciones de la biología y la medicina. Convenio relativo a los derechos humanos y la biomedicina. Oviedo, 4 de abril de 1997.

3. Daniels, N. "Justice, fair, procedens, and the goalds of medicine", Hasting Center Report 1996; 26 (nov-dec):10-12.

4. Lauzirika N. "Ios médicos de atención primaria, eslabón clave en los testamentos vitales". El Médico 2007; 16-XI-07:14-20 y 49.

5. Ley $41 / 2002$, de 14 de noviembre, básica reguladora de la autonomía del paciente y de derechos y obligaciones en materia de información y documentación clínica. BOE n² 274, de 15 de noviembre de 2002.

6. Sánchez Caro J. Configuración actual de la relación clínica. Rev Adm Sanit 2004; 2:433-61.

7. De Lorenzo y Montero R. Derechos y obligaciones de los pacientes. Madrid: Ed. Colex; 2003.

8. Declaración Universal de Derechos Humanos, de 10 de diciembre de 1948: preámbulo y artículos 12, 18-20, 25, 28 y 29.

9. Convenio para la protección de los derechos humanos y de las libertades fundamentales. Roma. 14 de noviembre de 1950: artículos 3, 4, 5, 8 y 9.

10. Simón Lorda P, Barrio JM, ¿Quién decidirá por mí? Madrid: Triacastela; 2004.

11. Cruceiro A. Las Voluntades Anticipadas de los enfermos (II). Jano 2001; LX: 718-719.

12. Santos Unamuno C. elmédicointeractivo. N $N^{\circ} 1977.26-28$ Enero 2008.

13. Perico, Giacomo: Problema di ética sanitaria. Milano: Ancora; 1985, $2^{a}(p)$.

14. Orgando Díaz B, García Pérez C, García Pérez MA, Jiménez Vázquez AM. "Contenidos éticos formales en la reciente legislación sanitaria”. Aten Primaria 2007; 39(5):267-72.

15. Asociación Derecho a Morir Dignamente. Voluntades Anticipadas / Testamento Vital. Disponible en: www.eutanasia.ws/textos/membreteTV.doc

16. Conferencia Episcopal Española. Testamento Vital. Disponible en: www.conferenciaepiscopal/servicios/testamento_vital. htm

17. Testigos de Jehová. Directriz o exoneración médica preliminar. Disponible en: www.anestesia.com.mx/art-17.html

18. Comité de Bioética de Cataluña. Testamento Vital. Disponible en: www.gtcv.org/leyes/textos_comite_bioetica.pdf

19. Organización Médica Colegial. Código de ética y Deontología médica. Madrid: OMC; 1999. Disponible en: www.cgcom. es/pdf/código.pdf

20. Ciurana Aparisi JC. "Ética en la consulta, las voluntades anticipadas". El Médico 2006; 31-III-06: 23-26.

21. Consejería de Sanidad de la Junta de Comunidades de Castilla La Mancha. Declaración de Voluntades Anticipadas (Testamento Vital). Dispoible en: www.jccm.es/sanidad/volprinci.html

22. Gracia D. "Ética y decisiones en el final de la vida". Eidon. Revista de la Fundación de Ciencias de la Salud 2006; 21:24-9. 